\title{
Resonance and Capture of Jupiter Comets
}

\author{
W.S. Koon*, M.W. Lo ${ }^{\dagger}$, J.E. Marsden* and S.D. Ross*
}

January, 2001

\begin{abstract}
A number of Jupiter family comets such as Oterma and Gehrels 3 make a rapid transition from heliocentric orbits outside the orbit of Jupiter to heliocentric orbits inside the orbit of Jupiter and vice versa. During this transition, the comet can be captured temporarily by Jupiter for one to several orbits around Jupiter. The interior heliocentric orbit is typically close to the 3:2 resonance while the exterior heliocentric orbit is near the 2:3 resonance.

An important feature of the dynamics of these comets is that during the transition, the orbit passes close to the libration points $L_{1}$ and $L_{2}$, two of the equilibrium points for the restricted three-body problem for the Sun-Jupiter system. Studying the libration point invariant manifold structures for $L_{1}$ and $L_{2}$ is a starting point for understanding the capture and resonance transition of these comets. For example, the recently discovered heteroclinic connection between pairs of unstable periodic orbits (one around the $L_{1}$ and the other around $L_{2}$ ) implies a complicated dynamics for comets in a certain energy range.

Furthermore, the stable and unstable invariant manifold "tubes" associated to libration point periodic orbits, of which the heteroclinic connections are a part, are phase space conduits transporting material to and from Jupiter and between the interior and exterior of Jupiter's orbit.
\end{abstract}

Keywords: comets, resonance, libration points, invariant manifolds, three-body problem

\section{Introduction}

A heteroclinic connection between periodic orbits about $L_{1}$ and $L_{2}$ was recently discovered by Koon, Lo, Marsden, and Ross [2000]. The existence of such a connection has important implications regarding the global dynamics of the three-body problem, and for the motion of bodies in the solar system experiencing such dynamics. The present paper summarizes earlier work on libration point orbits and their manifolds (see Conley [1968], McGehee [1969], Llibre, Martinez, and Simó [1985], and Koon, Lo, Marsden, and Ross [2000]) and applies the geometrical point of view to the comet resonance transition problem. The goal is to clearly state the qualitative dynamical picture that is forming, which any detailed investigation of transport between mean motion resonances must build upon. In Section 4, the particular case of transport between resonances interior and exterior to Jupiter's orbit is covered, following the example of the Jupiter family comet Oterma.

\footnotetext{
* Control and Dynamical Systems, California Institute of Technology, Pasadena, California, USA

$\dagger$ Navigation and Mission Design, Jet Propulsion Laboratory, Pasadena, California, USA 


\section{Jupiter Comets}

Resonance Transition in Comet Orbits. Some Jupiter comets such as Oterma and Gehrels 3 make a rapid transition from heliocentric orbits outside the orbit of Jupiter to orbits inside that of Jupiter and vice versa. During this transition, the comet may be captured temporarily by Jupiter for several orbits. The interior orbit is typically close to the 3:2 mean motion resonance while the exterior orbit is near the 2:3 resonance. See Figure 1(a). During the transition, the orbit passes close to the libration points $L_{1}$ and $L_{2}$, two of the equilibrium points (in a rotating frame) for the planar circular restricted 3-body problem (PCR3BP) for the Sun-Jupiter system.

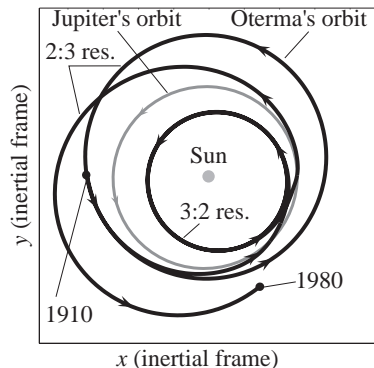

(a)

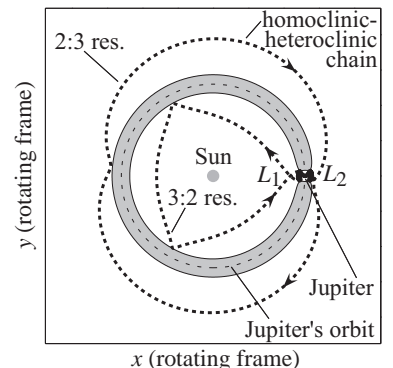

(b)

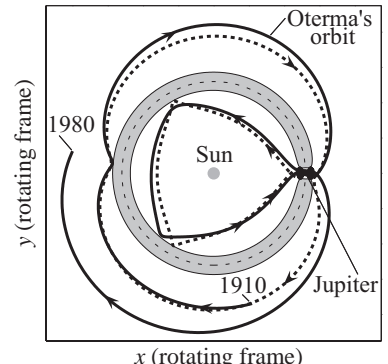

(c)

Figure 1. (a) Orbit of comet Oterma in Sun-centered inertial frame during time interval AD 1910-1980 (ecliptic projection). (b) The homoclinic-heteroclinic chain corresponding to the Jupiter comet Oterma. (c) The actual orbit of Oterma overlaying the chain.

The Relevance of Invariant Manifolds. Lo and Ross [1997] used the two degree of freedom PCR3BP as the underlying model for resonance transition and related the transition to invariant manifolds, noticing that the orbits of Oterma and Gehrels 3 (in the Sun-Jupiter rotating frame) closely follow the computed invariant manifolds of $L_{1}$ and $L_{2} \cdot{ }^{1}$ Koon, Lo, Marsden, and Ross [2000] developed this viewpoint along with another key ingredient, a heteroclinic connection between unstable periodic orbits around $L_{1}$ and $L_{2}$ with the same Jacobi constant (a multiple of the energy for the PCR3BP). The dynamical consequences of such an orbit are covered in great mathematical detail in that paper. Here, we focus on the study of exotic comet motion and resonance transtion in terms of the libration point invariant manifolds.

Heteroclinic Connections. A numerical demonstration is given in Koon, Lo, Marsden, and Ross [2000] of a heteroclinic connection between pairs of equal

\footnotetext{
1 Belbruno and B. Marsden [1997] considered the comet transitions using a different approach, the "fuzzy boundary" (or "weak stability boundary") concept, which they said "can be viewed as a higher-dimensional analogue of the collinear Lagrange points $L_{1}$ and $L_{2}$ of Jupiter." During their investigation however, they suggested that resonance transition "does not seem to occur in the planar circular restricted problem of two degrees of freedom."
} 
energy periodic orbits, one around $L_{1}$, the other around $L_{2}$. This heteroclinic connection augments the previously known homoclinic orbits associated with the $L_{1}$ and $L_{2}$ periodic orbits (see McGehee [1969]). Linking these heteroclinic connections and homoclinic orbits leads to dynamical homoclinicheteroclinic chains which form the backbone for temporary capture and rapid resonance transition of Jupiter comets. See Figure 1.

Existence and Construction of Transition Orbits. Koon, Lo, Marsden, and Ross [2000] prove the existence of a large class of interesting orbits in the neighborhood of a chain which a comet can follow in its rapid transition between the inside and outside of Jupiter's orbit via a Jupiter encounter. One can label orbits near a chain with an itinerary giving their past and future whereabouts, making their classification and manipulation possible. Furthermore, a systematic procedure for the numerical construction of orbits with prescribed itineraries has been developed using the stable and unstable invariant manifold tubes of $L_{1}$ and $L_{2}$ periodic orbits.

\section{A Few Key Features of the Three-Body Problem}

Planar Circular Restricted Three-Body Problem. The comets of interest are mostly heliocentric, and the perturbations of their motion away from Keplerian ellipses are dominated by Jupiter's gravitation. Moreover, their motion is nearly in Jupiter's orbital plane, and Jupiter's small eccentricity (0.0483) plays little role during the fast resonance transition (less than or equal to one Jupiter period in duration). The PCR3BP is therefore an adequate starting model for illuminating the essence of the resonance transition process.

The PCR3BP describes the motion of a body moving in the gravitational field of two main bodies that are moving in circles. The two main bodies we consider are the Sun and Jupiter. The total mass is normalized to 1; they are denoted $m_{S}=1-\mu$ and $m_{J}=\mu$, where $\mu=9.537 \times 10^{-4}$. The Sun and Jupiter rotate in the plane of their orbit in circles counterclockwise about their common center of mass and with angular velocity also normalized to 1 .

Equations of Motion. Choosing a rotating coordinate system so that the origin is at the center of mass, the Sun and Jupiter are on the $x$-axis at the points $(-\mu, 0)$ and $(1-\mu, 0)$ respectively-i.e., the distance from the Sun to Jupiter is normalized to be 1 . Let $(x, y)$ be the position of the comet in the plane, then the equations of motion in this rotating frame are:

$$
\ddot{x}-2 \dot{y}=\Omega_{x} \quad \ddot{y}+2 \dot{x}=\Omega_{y},
$$

where $\Omega=\frac{x^{2}+y^{2}}{2}+\frac{1-\mu}{r_{S}}+\frac{\mu}{r_{J}}$. Here, the subscripts of $\Omega$ denote partial differentiation in the variable. $r_{S}, r_{J}$ are the distances from the comet to the Sun and the Jupiter respectively. See Szebehely [1967] for the derivation. 
Energy Manifolds. These equations are autonomous and can be put into Hamiltonian form. They have an energy integral:

$$
E=\frac{1}{2}\left(\dot{x}^{2}+\dot{y}^{2}\right)-\Omega(x, y) .
$$

which is related to the Jacobi constant $C$ by $C=-2 E$. Energy manifolds are 3-dimensional surfaces foliating the 4-dimensional phase space. For fixed energy, Poincaré sections are 2-dimensional and therefore easily visualizable.

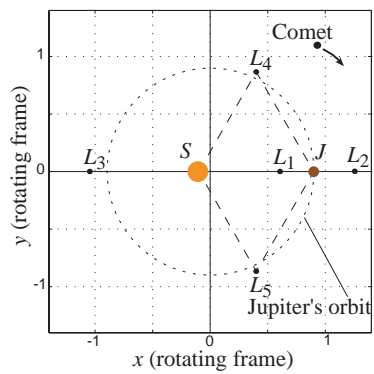

(a)

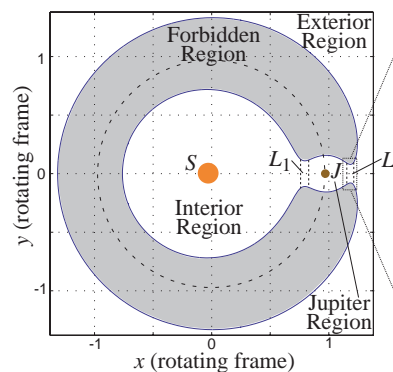

(b)

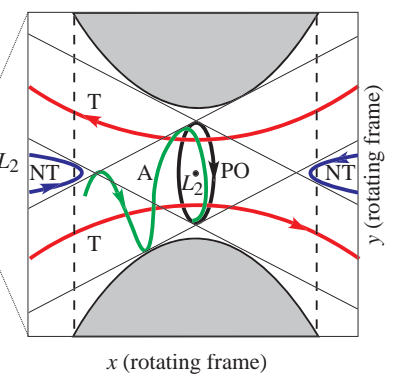

(c)

Figure 2. (a) Equilibrium points of the PCR3BP in the rotating frame. (b) Energetically forbidden region is gray " $C$ ". Hill's region (region in white), contains a "neck" about $L_{1}$ and $L_{2}$. (c) The flow in the region near $L_{2}$, showing a periodic orbit around $L_{2}$ (labeled PO), a typical asymptotic orbit winding onto the periodic orbit (A), two transit orbits (T) and two non-transit orbits (NT). A similar figure holds for the region around $L_{1}$.

Equilibrium Points. The PCR3BP has 3 collinear equilibrium (Lagrange) points which are unstable, but for the comets of interest, we examine only $L_{1}$ and $L_{2}$. See Figure 2(a). Eigenvalues of the linearized equations at $L_{1}$ and $L_{2}$ have one real and one imaginary pair, having a saddle $\times$ center structure.

Region of Possible Motion. The projection of the energy manifold onto the position space is the region in the $x y$-plane where the comet is energetically permitted to move around (known as the "Hill's region"). The forbidden region is the region that is not accessible for a given energy. See Figure 2(b).

Our main concern is the behavior of orbits whose energy is just above that of $L_{2}$, for which the Hill's region is a connected region with an interior region (inside Jupiter's orbit), exterior region (outside Jupiter's orbit), and a Jupiter (capture) region (bubble surrounding Jupiter). These regions are connected by "necks" about $L_{1}$ and $L_{2}$ and the comet can make transitions between the regions only through these necks. This equilibrium neck region and its relation to the global orbit structure is critical and is discussed next.

Four Types of Orbits in Equilibrium Regions. In each equilibrium region (one around $L_{1}$ and one around $L_{2}$ ), there exist 4 types of orbits (see Figure 
2(c)) as given in Conley [1968]: (1) an unstable periodic Lyapunov orbit; (2) 4 cylinders of asymptotic orbits that wind onto or off this period orbit, which form pieces of stable and unstable manifolds; (3) transit orbits which the comet must use to make a transition from one region to the other; and (4) nontransit orbits where the comet bounces back to its original region.
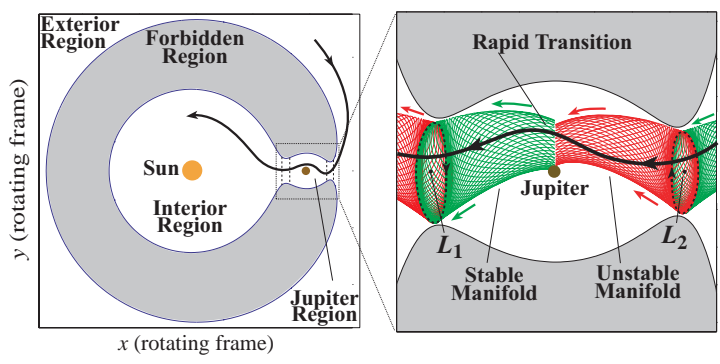

(a)

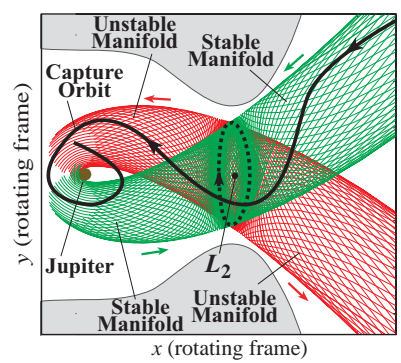

(b)

Figure 3. (a) Transit orbit from outside to inside Jupiter's orbit, passing by Jupiter. The tubes containing transit orbits (bounded by the cylindrical stable (lightly shaded) and unstable (darkly shaded) manifolds) intersect such that a transition is possible. (b) Orbit, beginning inside stable manifold tube in exterior region, is temporarily captured by Jupiter.

Invariant Manifolds as Separatrices. McGehee [1969] first observed that the asymptotic orbits are pieces of the 2-dimensional stable and unstable invariant manifold "tubes" associated to the Lyapunov orbit and they form the boundary between transit and nontransit orbits. The transit orbits, passing from one region to another, are those inside the cylindrical manifold tube. The nontransit orbits, which bounce back to their region of origin, are those outside the tube. Most importantly, to transit from outside Jupiter's orbit to inside (or vice versa), or get temporarily captured, a comet must be inside a tube of transit orbits, as in Figure 3. The invariant manifold tubes are global objects - they extend far beyond the vicinity of the equilibrium region, partitioning the energy manifold into regions of qualitatively different orbit behavior.

Numerical Computation of Invariant Manifolds. Periodic Lyapunov orbits can be computed using a high order analytic expansion (see Llibre, Martinez, and Simó [1985]). Their stable and unstable manifolds can be approximated as given in Parker and Chua [1989]. The basic idea is to linearize the equations of motion about the periodic orbit and then use the monodromy matrix provided by Floquet theory to generate a linear approximation of the periodic orbit's stable and unstable manifold. The linear approximation, in the form of a state vector, is numerically integrated in the nonlinear equations of motion to produce the approximation of the stable and unstable manifolds.

Rapid Transition Mechanism. The heart of the rapid transition mechanism from outside to inside Jupiter's orbit (or vice versa) is the intersection of 
transit orbit tubes. We can see the intersection clearly on a 2-dimensional Poincaré section in the 3-dimensional energy manifold. We take our section along a vertical line (parallel to the $y$-axis) through Jupiter as in Figure 4(a). Plotting $\dot{y}$ versus $y$ along this line, we see that the tube cross-sections are distorted circles (see Figure 4(b)). Upon magnification, it is clear the tubes indeed intersect (see Figure 4(c)).

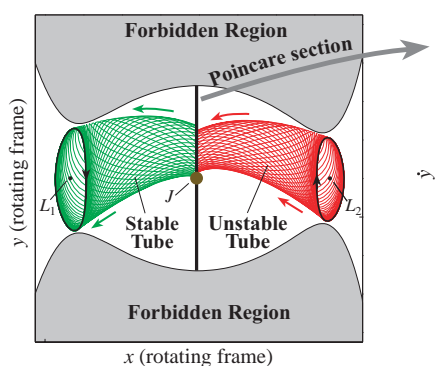

(a)

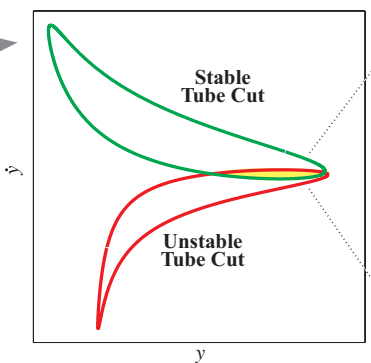

(b)

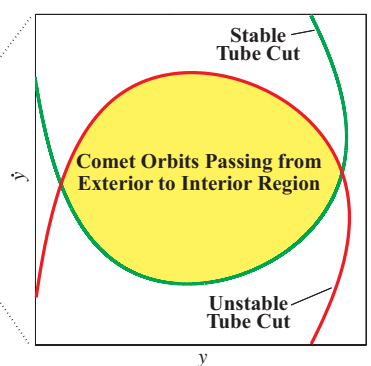

(c)

Figure 4. (a) Take a Poincaré section of the $L_{1}$ and $L_{2}$ periodic orbit invariant manifold tubes - a vertical line through Jupiter $(J)$. (b) Look at unstable tube cut for $L_{2}$ and stable tube cut for $L_{1}$. (c) A small portion of the tubes intersect - this set in the phase space contains the comet orbits which pass from the exterior to the interior region.

Any point within the region bounded by the curve corresponding to the stable tube cut is on an orbit that will go from the Jupiter region into the interior region. Similarly, a point within the unstable tube cut is on an orbit that came from the exterior region into the Jupiter region. A point inside the region bounded by the intersection of both curves (lightly shaded in Figure 4(c)) is on an orbit that makes the transition from the exterior region to the interior region, via the Jupiter region. The timescale for such a transition is short, less than one Jupiter period (Jupiter period $\approx 12$ years).

\section{Invariant Manifold Tubes and Resonance Transition}

Generic Transport Mechanism. This dynamical mechanism effecting transfer between the interior and exterior regions has not been previously recognized. It was previously believed that a third degree of freedom was necessary or that "Arnold diffusion" was somehow involved. But clearly, only the planar CR3BP is necessary. The dynamics and phase space geometry involved in the heteroclinic connection now provide a language with which to discuss and further explore resonance transition.

Transport Between Resonances. The dynamical channel discussed in the previous section is a generic transport mechanism connecting the interior and exterior regions. We now focus on the case of transport between resonances, 
and in particular, the rapid transport mechanism connecting inrerior and exterior mean motion resonances (e.g., the 3:2 and 2:3 Jupiter resonances). By numerically computing the connection between the interior and exterior resonances, we will obtain a deeper understanding of the mean motion resonance transition of actual Jupiter comets, such as Oterma.

Tube Location. In Figure 5, the location of the tubes is shown schematically. To perform an Oterma-like transition from outside to inside Jupiter's orbit, a comet orbit would begin inside the stable manifold tube of $L_{2}$ on the outside, then pass through the $L_{2}$ equilibrium region to the $L_{2}$ unstable manifold in the Jupiter region (as in Figure 3(a)). Intersecting the $L_{1}$ stable manifold tube in the Jupiter region, the trajectory would pass by $L_{1}$ into the interior region. Note, we will occasionally refer to the interior, Jupiter, and exterior regions with the letters $S, J$, and $X$, respectively.
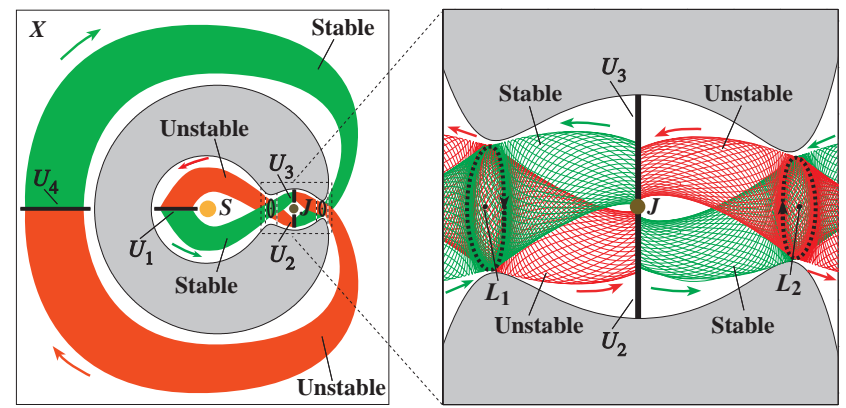

Figure 5. Location of $L_{1}$ and $L_{2}$ orbit invariant manifold tubes in position space (schematically). Stable manifolds are lightly shaded, unstable manifolds are darkly shaded. Location of Poincaré sections $\left(U_{1}, U_{2}, U_{3}\right.$, and $\left.U_{4}\right)$ also shown. Magnification of Jupiter region at right.

A comet orbit which circles the Sun once in the interior region of the rotating frame (as Oterma does as seen in Figure 1(c)) would have to be in a part of phase space where the $L_{1}$ stable and and unstable tubes intersect. We can see such an intersection along the $U_{1}$ section (see Figure 5).

Interior and Exterior Resonances. In Figure 6(a), we see a cross-section of the stable and unstable tubes of the $L_{1}$ Lyapunov orbit, transformed into Delaunay variables (see Szebehely [1967]). The vertical axis is an angular variable, thus we can identify the top and bottom boundaries. The background points reveal the mixed character of the interior region phase space for this energy surface: stable periodic and quasiperiodic tori "islands" embedded in a bounded chaotic "sea." The families of stable tori lie along strips of nearly constant semimajor axis, and correspond to mean motion resonances.

The first cuts of the stable and unstable tubes intersect at the 3:2 resonance. Any point inside the unstable curve is on an orbit which came from $J$ and any point inside the stable curve is on an orbit going toward $J$. Their in- 


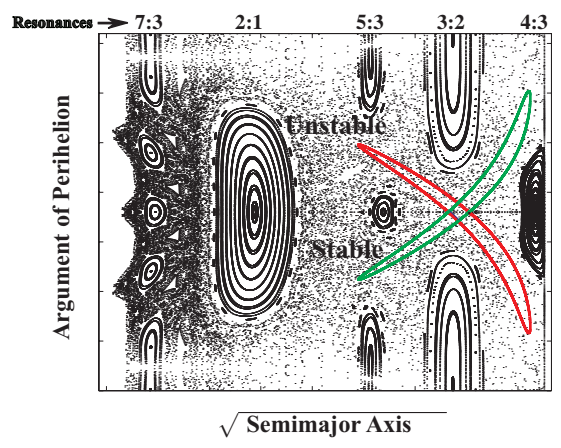

(a)

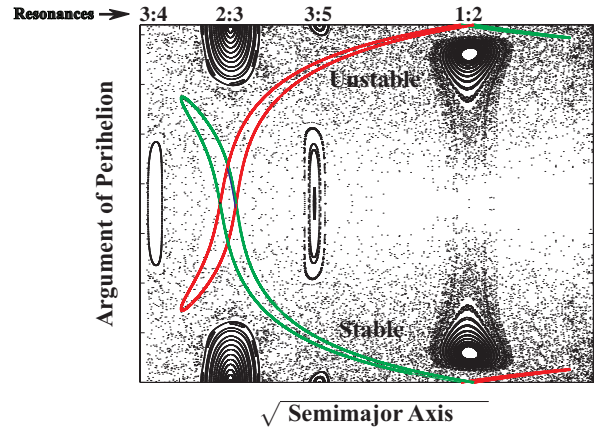

(b)

Figure 6. (a) The interior region $U_{1}$ Poincare section showing the first cuts of the stable and unstable manifold tubes of an $L_{1}$ Lyapunov orbit. Notice their intersection at the 3:2 resonance. (b) The exterior region $U_{4}$ Poincaré section showing the first cuts of the stable and unstable manifold tubes of an $L_{2}$ Lyapunov orbit. Notice their intersections at the 2:3 and 1:2 resonances.

tersection (the small diamond) contains all orbits that have come from $J$, have gone around the Sun once in the rotating frame, and will return to $J$. Because this intersection lies along the strip of 3:2 resonant orbits, we conclude that any comet which has an energy similar to Oterma's and which circles around the Sun once in the interior region must be in 3:2 resonance with Jupiter.

Similar to Figure 6(a) for the interior region, Figure 6(b) shows the first exterior region Poincaré cuts of the stable and unstable manifold tubes of an $L_{2}$ Lyapunov orbit with the $U_{4}$ section for the same energy, plotted using Delaunay variables. A similar mixed phase space structure is seen.

The stable and unstable tubes intersect at the region of the 2:3 resonance (the diamond). Any point inside the unstable curve is on an orbit which came from $J$ and any point inside the stable curve is on an orbit going toward $J$. Although there is another intersection at the 1:2 resonance, the cross-section of the tube is widest near the 2:3 resonance. These are close to canonical coordinates, thus the vicinity of the $2: 3$ resonance is the more important. Therefore, we expect any comet with this energy which just came from $J$ or is about to go to $J$, to be in 2:3 resonance with Jupiter. Their intersection (the diamond at the 2:3 resonance) contains the orbits that have come from $J$, circled the Sun once in the rotating frame, and will return to $J$.

Connection Between Resonances. These two resonances (the 3:2 in the interior and the 2:3 in the exterior) are dynamically linked for this energy via the intersection between tubes in the Jupiter region. In Figure 7(b), we reproduce Figure 4(c), showing the collection of orbits passing from the exterior to interior region. Superimposed upon this large shaded region are pieces of the image and pre-image of the 2:3 and 3:2 intersection diamonds, respec- 


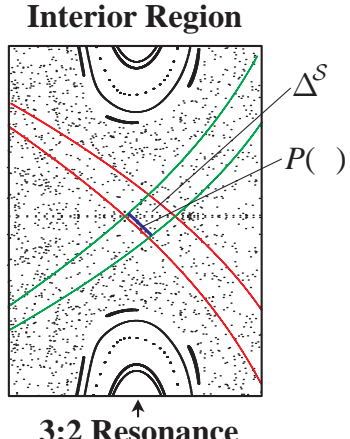

(a)

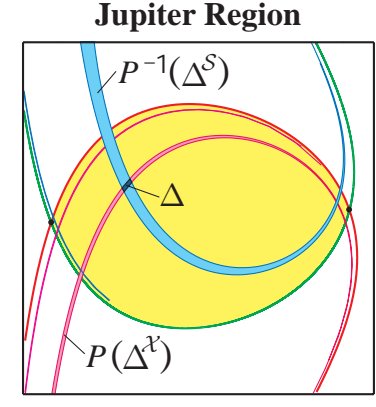

Resonance Connection

(b)
Exterior Region

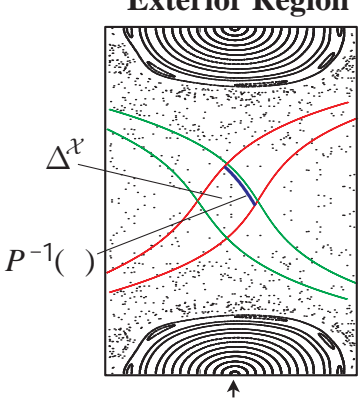

2:3 Resonance

(c)

Figure 7. (a) Interior region tube intersection $\Delta^{S}$. (b) The Jupiter region $U_{3}$ Poincaré section showing a portion of the image of $\Delta^{X}$ and the pre-image of $\Delta^{S}$. Notice their intersections, the largest of which is labeled $\Delta$. (c) Exterior region tube intersection $\Delta^{X}$.

tively ( $\Delta^{X}$ in the exterior region and $\Delta^{S}$ in the interior region, respectively). The diamonds are mapped to highly stretched and folded strips $P\left(\Delta^{X}\right)$ and $P^{-1}\left(\Delta^{S}\right)$ in the Jupiter region. Here, $P$ denotes the Poincaré map connecting the sections $U_{1}, U_{2}, U_{3}$, and $U_{4}$.

Note that $P\left(\Delta^{X}\right)$ and $P^{-1}\left(\Delta^{S}\right)$ intersect; the largest of these intersections is labeled $\Delta$. The image and pre-image of $\Delta$ are small strips in the interior and exterior regions respectively (see Figures 7(a) and (c)). This is an open set in the energy surface which dynamically links the 3:2 and 2:3 resonances via the Jupiter region. One can pick any point inside the strip $\Delta$ and integrate it forward and backward, generating an Oterma-like transition from the 2:3 to the 3:2 resonance. See Figure 8 and compare with Figure 1.

We conclude that $L_{1}$ and $L_{2}$ invariant manifold tubes and their intersections lead to the resonance transition exhibited by comets like Oterma. We expect that Oterma executes a transition near the full model analogue of this

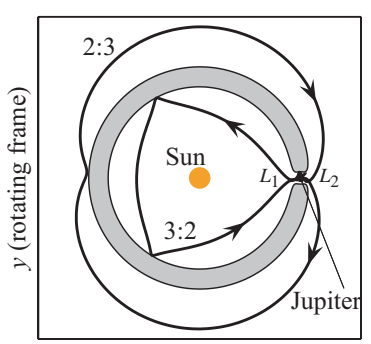

$x$ (rotating frame)

(a)

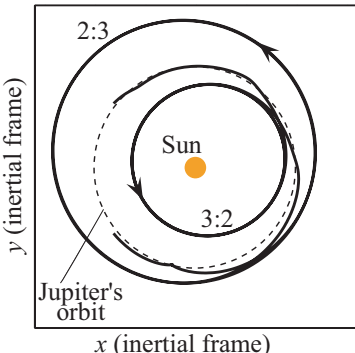

(b)

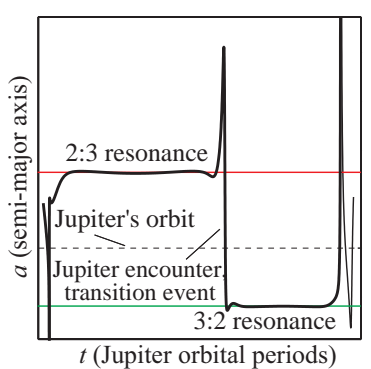

(c)

Figure 8. (a) An Oterma-like resonance transition in the rotating frame. (b) The same orbit in the heliocentric inertial frame. (c) Plot of semimajor axis versus time for the same orbit. Important mean motion resonances 3:2 and 2:3 are also shown for comparison. 
dynamical channel. For example, the $L_{1}$ and $L_{2}$ invariant manifold structures in the three degree of freedom system are important for comets of similar energy like Helin-Roman-Crockett (see Howell, Marchand, and Lo [2000]).

Other Resonance Connections. A similar resonance connection should exist for all nearby energies, as confirmed by numerical experiment. We have seen a link between only first order resonances $(p: q$, where $|p-q|=1)$ because we looked only at the first Poincaré cut of the tubes on our chosen surface in this study. Looking at cuts beyond the first reveals transitions between higher order resonances. In addition, higher energies have "larger," more dispersive tubes, which have more intersections for a given cut number.

\section{Conclusions}

We have applied dynamical systems techniques developed in Koon, Lo, Marsden, and Ross [2000] to the problem of resonance transitions and capture of Jupiter comets with energies near Oterma's. The fundamental mechanism is the rapid transport between the outside and inside of Jupiter's orbit via $L_{1}$ and $L_{2}$ periodic orbit invariant manifold tubes containing transit orbits. This mechanism provides a starting point for understanding the transport between mean motion resonances in more complicated models. Furthermore, the invariant manifold structures associated with $L_{1}$ and $L_{2}$ periodic orbits may prove valuable for understanding transport throughout the solar system.

\section{References}

Belbruno, E. and B. Marsden. Resonance Hopping in Comets. The Astronomical Journal. 113(4): 1433-1444, 1997.

Conley, C. Low Energy Transit Orbits in the Restricted Three-Body Problem. SIAM J. Appl. Math. 16: 732-746, 1968.

Howell, K.C., B.G. Marchand, and M.W. Lo. Temporary Satellite Capture of Short-Period Jupiter Family Comets from the Perspective of Dynamical Systems. AAS/AIAA Space Flight Mechanics Meeting, Clearwater, Florida, USA. Paper No. 00-155, 2000.

Koon, W.S., M.W. Lo, J.E. Marsden and S.D. Ross. Heteroclinic connections between periodic orbits and resonance transitions in celestial mechanics. Chaos 10(2): 427-469, 2000.

Llibre, J., R. Martinez, and C. Simó. Transversality of the Invariant Manifolds Associated to the Lyapunov Family of Periodic Orbits Near L2 in the Restricted Three-Body Problem. Journal of Differential Equations 58: 104-156, 1985.

Lo, M. and S. Ross. SURFing the Solar System: Invariant Manifolds and the Dynamics of the Solar System. JPL IOM 312/97, 2-4, 1997.

McGehee, R. Some Homoclinic Orbits for the Restricted Three Body Problem. Ph.D. Thesis, University of Wisconsin, Madison, Wisconsin, USA, 1969.

Parker, T.S. and L.O. Chua. Practical Numerical Algorithms for Chaotic Systems, SpringerVerlag, New York, 1989.

Szebehely, V. Theory of Orbits, Academic Press, New York, 1967. 